\title{
Equid herpesvirus type 1 (EHV-1) disrupts actin cytoskeleton during productive infection in equine leukocytes
}

\author{
Z. Drebert ${ }^{1,2}$, A. Golke1, J. Cymerys ${ }^{1}$, A. Słońska ${ }^{1,3}$, A. Chmielewska ${ }^{1}$, \\ A. Tucholska', M.W. Banbura' ${ }^{1}$ \\ ${ }^{1}$ Division of Microbiology, Department of Preclinical Sciences, Faculty of Veterinary Medicine, \\ Warsaw University of Life Sciences (SGGW), Ciszewskiego 8, 02-786 Warsaw, Poland \\ ${ }^{2}$ Laboratory of Experimental Cancer Research, Department of Radiation Therapy \\ and Experimental Cancer Research, Ghent University, UZ 1P7, De Pintelaan 185, B-9000 Ghent, Belgium \\ ${ }^{3}$ Division of Physiology, Department of Physiological Sciences, Faculty of Veterinary Medicine, \\ Warsaw University of Life Sciences (SGGW), Ciszewskiego 8, 02-786 Warsaw, Poland
}

\begin{abstract}
Equid herpesvirus type 1 (EHV-1) is a prevalent causative agent of equine diseases worldwide. After primary replication in the respiratory epithelium the virus disseminates systemically through a peripheral blood mononuclear cell (PBMC)-associated viraemia. EHV-1 is the only alphaherpesvirus known so far which is capable of establishing latent infection not only in neurons but also in immune system cells (mainly in lymphocytes and macrophages). Since leukocytes are not the target cells for viral replication but are used to transport EHV-1 to the internal organs, the question remains how the virus avoids the immune response and whether it could potentially be associated with virus-induced cytoskeletal rearrangements. Therefore, the aim of this study was to investigate the progress of EHV-1 replication in leukocytes stimulated by phytohemagglutinin and the impact of EHV-1 infection on the actin cytoskeleton. Using the real-time PCR method we evaluated the quantity of viral DNA from samples collected at indicated time points post infection. In order to examine possible changes in actin cytoskeleton organization due to EHV-1 infection, we performed immunofluorescent staining using TRITC-phalloidin conjugate. The results showed that EHV-1 replicates in leukocytes at a restricted level but with the accompaniment of chromatin degradation. Simultaneously, infection with EHV-1 caused disruption of the actin cytoskeleton; this was particularly apparent in further stages of infection. Disruption of the actin cytoskeleton may lead to the limited release of the virus from the cells, but may be also beneficial for the virus, since at the same time it potentially impairs the immune function of leukocytes.
\end{abstract}

Key words: EHV-1, leukocytes, actin cytoskeleton 


\section{Introduction}

Equine herpesvirus type 1 (EHV-1), a member of the Alphaherpesvirinae subfamily, is a world spread pathogen of major economic importance in horses, causing mild respiratory diseases, abortion and neurological disorders (Patel and Heldens 2005). Extensive cell-associated viraemia is detected from 4-6 days after EHV-1 infection and lasts until 9-14 days after infection (Gibson et al. 1992). Viraemia may occur in the presence of virus-neutralizing antibodies (Mumford et al. 1987). EHV-1 has the ability to establish latent infection in leukocytes and trigeminal ganglion neurons, which play a significant role in the epidemiology of EHV-1-caused abortions (Baxi et al. 1996). One of the major problems associated with research on the replication of EHV-1 is the choice of the experimental model. For obvious reasons, the use of a natural host is very limited and therefore alternative solutions are being sought. One of the previously presented experimental models is the culture of freshly isolated, equine peripheral blood mononuclear cells (PBMC) (van Der Meulen et al. 2000). It has been demonstrated that in vitro mitogen stimulation of leukocytes enhances EHV-1 replication. Activation of T-lymphocytes may be an important pathogenic feature during an EHV-1 infection (McCulloch et al. 1993). Blastic transformation of lymphocytes, induced in vitro by mitogens or induced in vivo during an EHV-1 infection, most likely provides a signal for the virus to start its replication (van Der Meulen et al. 2000). In the present study we have investigated the dynamic of EHV-1 replication in mitogen stimulated equine leukocytes and reorganization of the actin cytoskeleton, which can be utilized by viruses during entry, replication or egress from the cells. The actin cytoskeleton is a dynamic structure that plays a crucial role in many cellular processes. Various herpesviruses interact with the cell cytoskeleton throughout their replication cycle, which may cause either actin polymerization or fragmentation of filaments (Döhner and Sodeik 2004, Roberts and Baines 2011, Taylor et al. 2011). The influence of EHV-1 on the cytoskeleton was previously examined in Vero cells (simian kidney cells), ED cell culture (equine dermal cells) and primary murine neurons. It has been shown that changes in the arrangement of the cytoskeleton depend on the origin of the cells (Walter and Nowotny 1999, Turowska et al. 2007, Turowska et al. 2010, Słońska et al. 2014). Therefore, the aim of this study was to investigate the progress of EHV-1 replication in equine leukocytes in relation to its impact on the actin cytoskeleton. This approach is particularly justified in relation to the fact that intact cytoskeleton structures are vital to maintain the immune function of these cells.

\section{Materials and Methods}

\section{Cell culture and viruses}

The cell culture was established by isolating the fraction of peripheral blood lymphocytes from horses free of EHV-1 infection as described previously (Bańbura et al. 2000). The cells were cultured in LymphoGrow medium containing phytohemagglutinin (Cytogen) and after 24 hours of incubation at $37^{\circ} \mathrm{C}$ with $5 \% \mathrm{CO}^{2}$ the cell cultures were infected with approximately $10^{3} \mathrm{CCID}_{50} / \mathrm{ml}$ of a field strain of EHV-1 (Jan-E), isolated from an aborted foetus (Janów Podlaski stud, Poland).

\section{Quantitative real-time PCR}

Viral DNA was isolated from samples collected after 2, 4, 8, 24, 48, 72 hours and 7 days post infection (p.i.), separately from leukocytes and cell culture medium. The quantity of the EHV-1 DNA in all samples was estimated using real-time PCR (qPCR) with a fluorescent TaqMan probe. For amplification of viral DNA, primers specific for the glycoprotein B $(\mathrm{gB})$ gene were used. Serial dilutions of Jan-E strain DNA were used as reaction standards. Uninfected PMBCs were used as a negative control. Tests were run on a LightCycler 2.0 instrument (Roche Diagnostics) using an in-house quantitative method as described previously (Dzieciątkowski et al. 2009, Cymerys et al. 2010).

\section{Immunofluorescent staining}

For immunofluorescent staining, cells were plated onto laminin-coated coverslips. After 2, 4, 8, 24, 48 and $72 \mathrm{~h}$ p.i. the cells were fixed in $3.7 \%$ paraformaldehyde/PBS (Sigma Chemicals) for $30 \mathrm{~min}$ at room temperature. The cells were permeabilized in $0.5 \%$ Tween/PBS for $5 \mathrm{~min}$, washed in PBS and blocked with PBS containing $1 \%$ bovine serum albumin (BSA) (Sigma Chemicals). Filament structures of actin were visualized using TRITC-phalloidin conjugate (500 $\mathrm{ng} / \mathrm{ml}$; Sigma Chemicals). Polyclonal antiserum EHV-1/ERV conjugated to FITC (VMRD, Inc.) was used to detect viral antigens and the cell nuclei were stained with Bisbenzimidine/Hoechst 33258 according to the manufacturerAs recommendations. Cells were examined under a BX-60 Olympus microscope using Cell^ $\mathrm{F}$ software. 
A

B
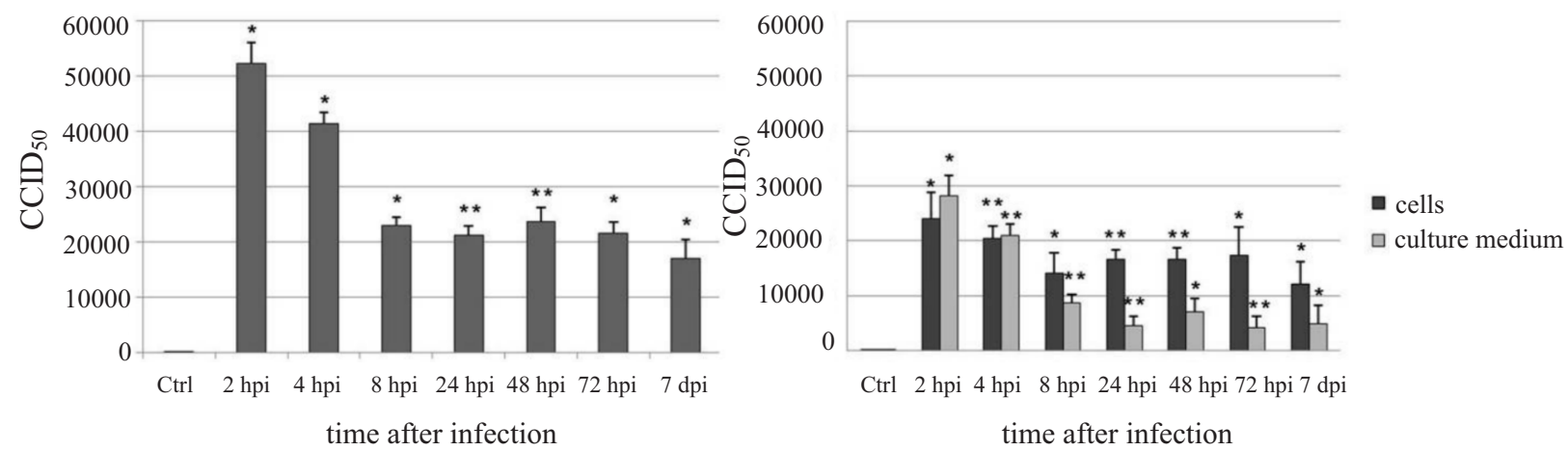

Fig. 1. Real-time PCR quantification of EHV-1 DNA in equine leukocytes. Dynamics of EHV-1 replication in leukocytes from $2 \mathrm{~h}$ p.i. to 7 days p.i. (A) Comparison of amounts of EHV-1 DNA in leukocytes and cell culture medium in samples collected from $2 \mathrm{~h}$ p.i. to 7 days p.i. (B) Differences in mean values in relation to control uninfected leukocytes were interpreted as significant at $\mathrm{P}<0.05\left(^{*}\right)$ and highly significant at $\mathrm{P}<0.01(* *)$.
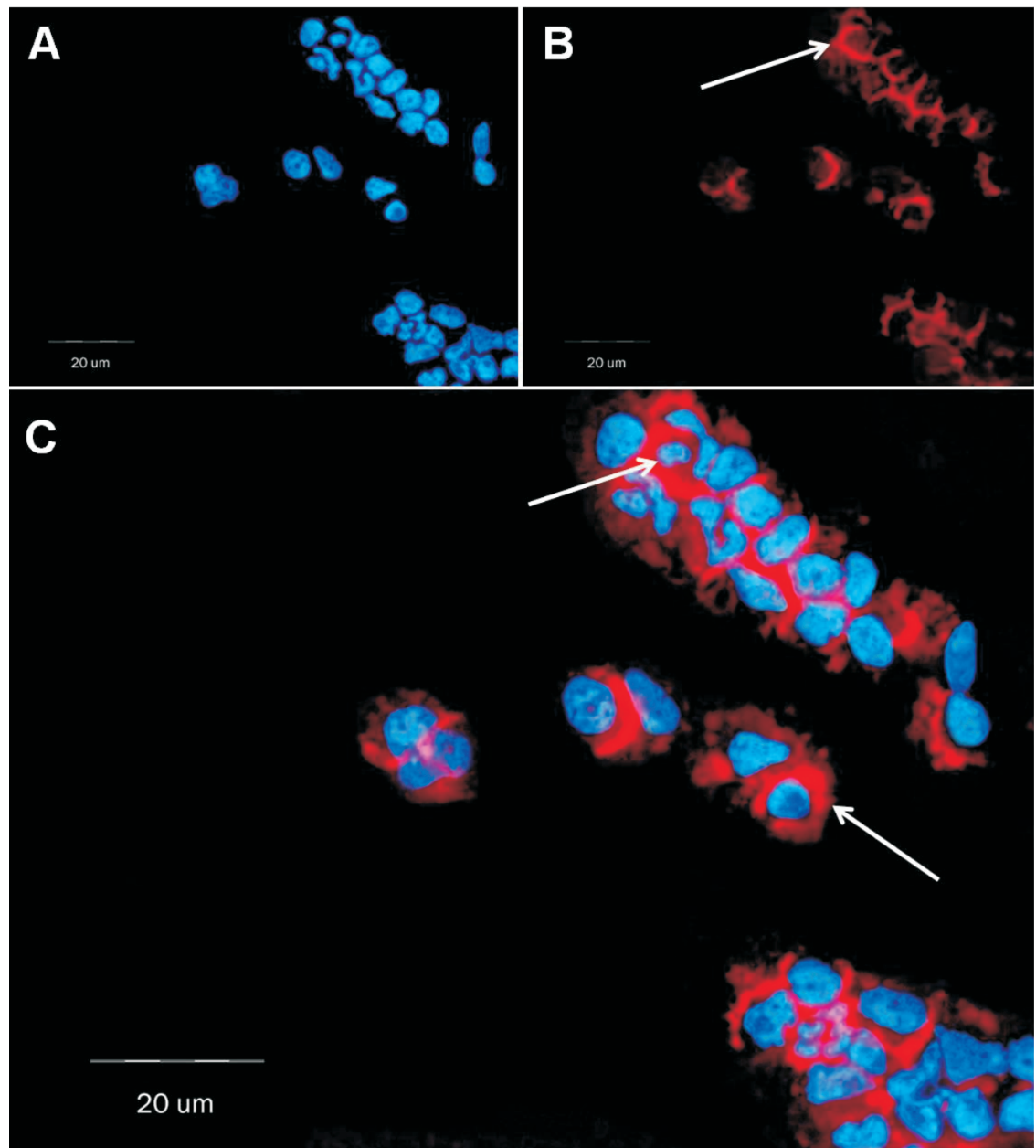

Fig. 2. Immunofluorescence image of uninfected leukocytes. Staining for actin (B) (red fluorescence - arrows), nuclei (A) (blue fluorescence), (C) imposition of fluorescence channels. Microscope magnification 40x.

\section{Statistical analysis}

All experiments were performed in triplicate and each analysis was repeated at least twice. Differences in mean values in relation to control (uninfected leukocytes) were analyzed using Student's t-test (STATISTICA software - Version 10). Statistical differ-ences were interpreted as significant at $\mathrm{P}<0.05\left(^{*}\right)$ and highly significant at $\mathrm{P}<0.01(* *)$. 

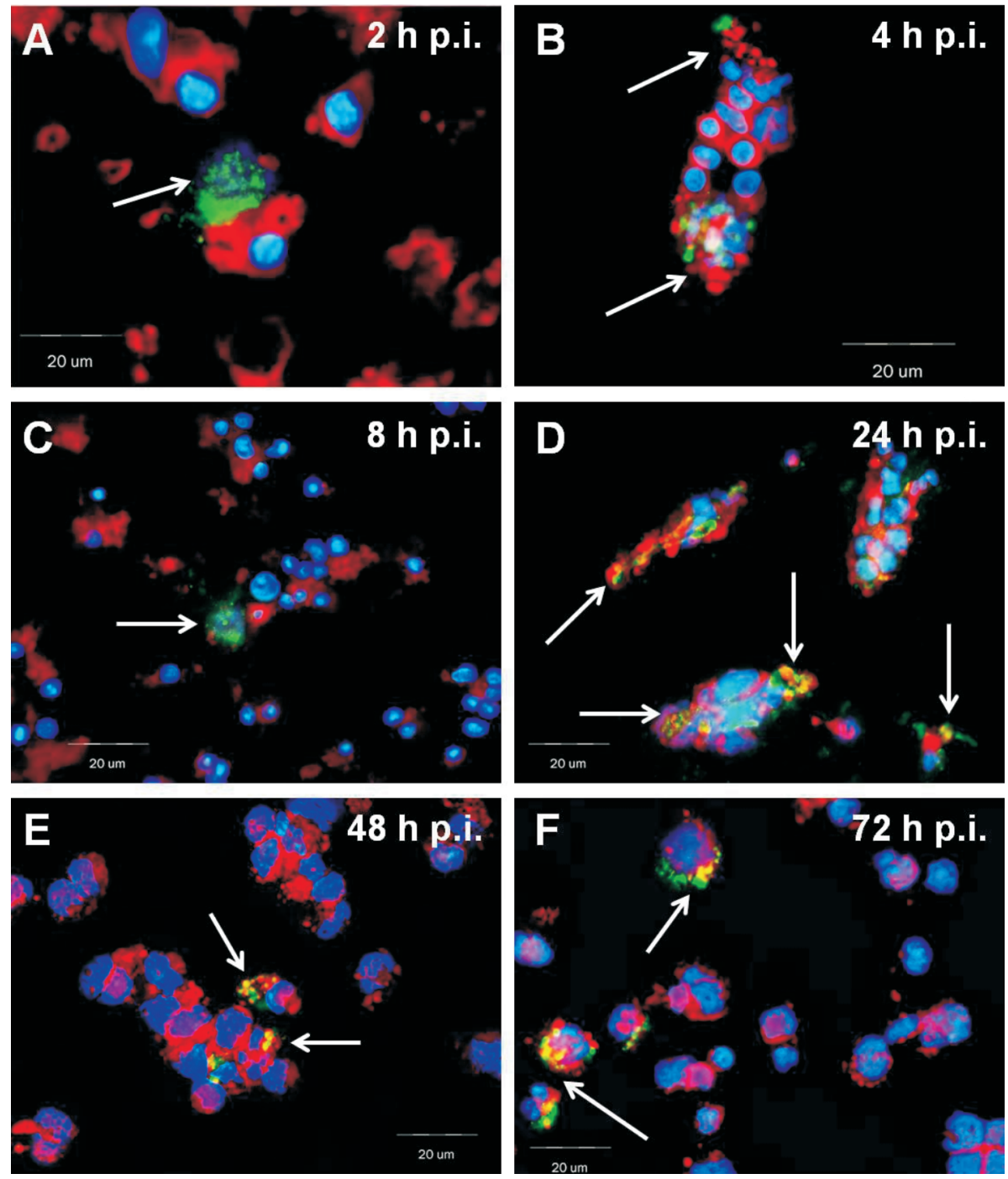

Fig. 3. Immunofluorescence image of leukocytes infected with Jan-E strain of EHV-1. Staining for actin (red fluorescence), nuclei (blue fluorescence), EHV-1 antigen (green fluorescence). Microscope magnification 40x.

\section{Results}

In this paper we present preliminary results concerning the dynamic of EHV-1 replication in mitogen-stimulated leukocytes and its influence on the organization of the actin cytoskeleton. The PCR results indicated an initial predomination of viral DNA in culture medium in comparison to cells. However, in the further stages of infection variation of the level of viral DNA in cells and in the culture medium was observed. From 8 h.p.i. up to 7 d.p.i. the amount of viral DNA was maintained at a higher level in cells than in the culture medium (Fig. 1B). Microscopy analysis confirmed the presence of EHV-1 antigen in the infected cells at all the examined time points. From 2 h.p.i. to 8 h.p.i. the signal was emitted from the nuclei and was also accompanied by the destruction of chromatin (Fig. 3A,B,C). However, in the subsequent stages of infection the signal was emitted from cytoplasmic regions, mainly close to the nuclei (Fig. 3E,F - arrows). We also found the florescence to be most intense in the cells that were already damaged. In the control (uninfected cells) actin microfilaments were observed within the cytoplasm with the densest distribution in the peripheral regions of the plasma membrane (Fig. 2B,C - arrows). During infection with EHV-1 the organization of the actin cytoskeleton was altered compared to uninfected control cells as early as $4 \mathrm{~h}$ p.i. (Fig. 3B). In further stages of infection, first a general depolymerization of actin (Fig. 3C) and subsequently significant changes in the organization of the actin cytoskeleton were observed. Actin was not organized in a network of fibers but formed spherical structures distributed in the whole area of the cell; this was accompanied by the accumulation of viral antigen around the nuclei (Fig. 3D,E,F - arrows). 


\section{Discussion}

Results indicated that, although EHV-1 replicates in leukocytes at a restricted level, it is not effectively released from the cells. Since actin plays a crucial role in intracellular transport, which is critical for viral replication and egress, a limited virus release from the cells may result from observed changes in the actin cytoskeleton. However, it should also be emphasized that leukocytes are not specific target cells for EHV-1 replication which, after primary replication in the respiratory epithelium, uses leukocytes to spread to internal organs. These are also the cells in which the virus establishes latency (Baxi et al. 1996). As was previously noted, the effect of viral infection on the cytoskeleton structure may vary depending on the cell type (Turowska et al. 2010, Słońska et al. 2014). It has been shown that the Jan-E strain induced polymerization of F-actin in Vero cells but not in ED cells (Turowska et al. 2010). In primary murine neurons the Jan-E strain did not disrupt the actin cytoskeleton, but caused a rearrangement of actin distribution and induced the formation of actin-containing cell projections, which stretched from cell to cell (Słońska et al. 2014). Presumably, EHV-1 utilizes actin filaments in leukocytes at the initial stage of infection, as fluorescent images confirmed that the virus is able to reach the site of replication. Nevertheless, it is unclear whether an intact actin cytoskeleton is essential for EHV-1 replication. Our previous studies on primary murine neurons with cytoskeletal inhibitors indicated that the level of replication of the Jan-E strain was similar in neurons with a damaged and with an intact actin cytoskeleton as well as nucleo-cytoplasmic transport receptors (Słońska et al. 2013, Słońska et al. 2014). However, it is worth mentioning that the role of the various components of the cytoskeleton in the replication cycle of a particular virus may differ depending on the type of infected cells. In the case of the Epstein-Barr virus (EBV) the role of actin in its transport differs significantly in B lymphocytes and in epithelial cells. It was observed that the virus enters these cells using different mechanisms. Moreover, all viral particles entering $\mathrm{B}$ cells remain stable for at least $8 \mathrm{~h}$, while up to $80 \%$ of viral particles entering epithelial cells are degraded. Furthermore, transport of the virus to the nucleus requires involvement of the actin cytoskeleton only in epithelial cells (Valencia and Hutt-Fletcher 2012). However, interactions between viruses and the host cytoskeleton do not have to be connected with virus trafficking within the infected cell. In leukocytes, virus-induced cytoskeletal changes may lead to an impairment of cell functions, as it was proven that involvement of the actin cytoskeleton is essential in molecular organization at the immunological synapse for- med between $\mathrm{T}$ lymphocytes and antigen-presenting cells (Valitutti et al. 1995, Campi et al. 2005). It has been demonstrated that human immunodeficiency virus (HIV-1) infection of $\mathrm{T}$ lymphocytes affects the cytoskeleton, impairing the generation and function of immunological synapses, in order to ensure cell survival, virus replication and spread (Soares et al. 2013).

In conclusion, our preliminary results indicate that EHV-1 enters the cell and replicates in leukocytes at a limited level, simultaneously causing disruption of the actin cytoskeleton and this is particularly apparent in further stages of infection. Therefore, it is tempting to speculate that these changes may have an impact on the limited release of the virus from the cells. Considering that rearrangements of the actin cytoskeleton caused by EHV-1 infection may differ depending on the strain used for infection, further studies with other EHV-1 strains are required. Moreover, in order to determine whether the viral infection influences only the actin cytoskeleton structure or whether it also causes changes in F-actin protein level, it would also be necessary to perform a quantitative evaluation based on scanning cytometry, measuring F-actin-related fluorescence intensity. Additionally, as the interplay between different cytoskeleton elements has still not been examined in the case of infection of leukocytes with EHV-1, further studies concerning microtubules, motor proteins and intermediate filaments are also required.

\section{Acknowledgement}

This work was supported by a grant from the Polish Ministry of Science and Higher Education, No. N308 309633.

\section{References}

Bańbura M, Chmielewska A, Tucholska A, Malicki K (2000) Occurrence of equine herpes virus type-1 (EHV-1) - specific DNA sequences in peripheral blood leukocytes of horses. Medycyna Wet 56: 521-523.

Baxi MK, Borchers K, Bartels T, Schellenbach A, Baxi S, Field HJ (1996) Molecular Studies of the Acute Infection, Latency and Reactivation of Equine Herpesvirus-1 (EHV-1) in the Mouse Model. Virus Res 40: 33-45.

Campi G, Varma R, Dustin ML (2005) Actin and agonist MHC-peptide complex-dependent $\mathrm{T}$ cell receptor microclusters as scaffolds for signaling. J Exp Med 202: 1031-1036.

Cymerys J, Dzieciątkowski T, Słońska A, Bierla J, Tucholska A, Chmielewska A, Golke A, Bańbura MW (2010) Equine herpesvirus type $1(\mathrm{EHV}-1)$ replication in primary murine neurons culture. Pol J Vet Sci 13: 701-708.

Döhner K, Sodeik B (2004) The role of the cytoskeleton 
during viral infection. Curr Top Microbiol Immunol 285: 67-108.

Dzieciątkowski T, Przybylski M, Cymerys J, Turowska A, Chmielewska A, Tucholska A, Bańbura MW (2009) Equine herpesvirus type 1 quantification in different types of samples by a real-time PCR. Pol J Vet Sci 12: 311-315.

Gibson JS, O'Neill T, Thackray A, Hannant D, Field HJ (1992) Serological responses of specific pathogen-free foals to equine herpesvirus-1: primary and secondary infection and reactivation. Vet Microbiol 32: 199-214.

McCulloch J, Williamson SA, Powis SJ, Edington N (1993) The effect of EHV-1 infection upon circulating leucocyte populations in the natural equine host. Vet Microbiol 37: 147-161.

Mumford JA, Rossdale PD, Jessett DM, Gann SJ, Ousey J, Cook RF (1987) Serological and virological investigations of an equid herpesvirus 1 (EHV-1) abortion storm on a stud farm in 1985. J Reprod Fertil Suppl 35: 509-518.

Patel JR, Heldens J (2005) Equine herpesviruses 1 (EHV-1) and 4 (EHV-4) - epidemiology, disease and immunoprophylaxis: a brief review. Vet J 170: 14-23.

Roberts KL, Baines JD (2011) Actin in herpesvirus infection. Viruses 3: 336-346.

Słońska A, Cymerys J, Skwarska J, Golke A, Bańbura MW (2013) Influence of importin alpha/beta and exportin 1 on equine herpesvirus type 1 (EHV-1) replication in primary murine neurons. Pol J Vet Sci 16: 749-751.

Słońska A, Cymerys J, Godlewski MM, Dzieciątkowski T, Tucholska A, Chmielewska A, Golke A, Bańbura MW (2014) Equine herpesvirus type 1 (EHV-1)-induced rearrangements of actin filaments in productively infected primary murine neurons. Arch Virol 159: 1341-1349.
Soares H, Lasserre R, Alcover A (2013) Orchestrating cytoskeleton and intracellular vesicle traffic to build functional immunological synapses. Immunol Rev 256: 118-132.

Taylor MP, Koyuncu OO, Enquist LW (2011) Subversion of the actin cytoskeleton during viral infection. Nat Rev Microbiol 9: 427-439.

Turowska A, Chmielewska A, Tucholska A, Banbura WM (2007) Influence of equine herpesvirus type 1 (EHV-1) infection on actin cytoskeleton. Medycyna Wet 63: 80-83.

Turowska A, Paj k B, Godlewski MM, Dzieci tkowski T, Chmielewska A, Tucholska A, Banbura M (2010) Opposite effects of two different strains of equine herpesvirus 1 infection on cytoskeleton composition in equine dermal ED and African green monkey kidney Vero cell lines: application of scanning cytometry and confocal-microscopy-based image analysis in a quantitative study. Arch Virol 155: 733-743.

Valencia SM, Hutt-Fletcher LM (2012) Important but differential roles for actin in trafficking of Epstein-Barr virus in B cells and epithelial cells. J Virol 86: 2-10.

Valitutti S, Dessing M, Aktories K, Gallati H, Lanzavecchia A (1995) Sustained signaling leading to $T$ cell activation results from prolonged $\mathrm{T}$ cell receptor occupancy. Role of T cell actin cytoskeleton. J Exp Med 181: 577-584.

van Der Meulen KM, Nauwynck HJ, Buddaert W, Pensaert MB (2000) Replication of equine herpesvirus type 1 in freshly isolated equine peripheral blood mononuclear cells and changes in susceptibility following mitogen stimulation. J Gen Virol 81: 21-25.

Walter I, Nowotny N (1999) Equine herpes virus type 1 (EHV-1) infection induces alterations in the cytoskeleton of Vero cells but not apoptosis. Arch Virol 144: 1827-1836. 